\title{
Argon, silicon and iron abundances in the damped Ly- $\alpha$ system I Zw 18
}

\author{
S. A. Levshakov ${ }^{1}$, W. H. Kegel ${ }^{2}$, and I. I. Agafonova ${ }^{1}$ \\ 1 Department of Theoretical Astrophysics, Ioffe Physico-Technical Institute, 194021 St. Petersburg, Russia \\ e-mail: lev@astro.ioffe.rssi.ru \\ e-mail: ira@astro.ioffe.rssi.ru \\ 2 Insitut für Theoretische Physik der Universität Frankfurt am Main, 60054 Frankfurt/Main 11, Germany \\ e-mail: kegel@astro.uni-frankfurt.de
}

Received 28 November 2000 / Accepted 1 May 2001

\begin{abstract}
We show how the ratio of the abundances of Ar, Si and Fe as derived from FUSE absorption spectra of I Zw 18 are affected by large-scale stochastic velocity fields (mesoturbulence). 21-cm observations with high spatial resolution show the presence of velocity fields with large correlation lengths. FUSE observations were performed with a large entrance aperture which fully covered the galaxy. This means that the observed profiles are averaged over the full body of I $\mathrm{Zw}$ 18. Taking this into account, we show that the observations are consistent with the abundance ratios $[\alpha$-chain/Fe] being similar to those derived from Galactic metal-poor stars. Using a mesoturbulent approach, we found that the observed profiles can be reproduced well with $[\mathrm{Fe} / \mathrm{H}]=-2.4 \pm 0.1$, and $[\mathrm{Si}, \mathrm{Ar} / \mathrm{Fe}]=0.7 \pm 0.1$.
\end{abstract}

Key words. line: formation - line: profiles - galaxies: individual: I Zw 18

\section{Introduction}

The blue compact galaxy (BCG) I Zw 18 (Mrk 116) has been an intensively studied object for the last three decades since the first spectroscopic observations by Zwicky (1966). I Zw 18 shows a powerful and recent burst of star formation which makes this galaxy an attractive target for studies of the history of star formation. For example, recent observations revealed two stellar populations in I Zw 18: 10-20 Myr red supergiants and 0.1-5 Gyr asymptotic giant branch stars (Östlin 2000).

Amongst BCGs, I $\mathrm{Zw}_{\mathrm{w}} 18$ shows the lowest oxygen abundance: the northwest (NW) and the southeast (SE) bright $\mathrm{H}$ II regions yield $(\mathrm{O} / \mathrm{H})^{3}=-4.83 \pm 0.03$ and $-4.82 \pm 0.03$, respectively (Izotov et al. 1999). Relative to solar values, one finds $[\mathrm{O} / \mathrm{H}]^{4}=-1.70 \pm 0.08$, i.e. $Z / Z_{\odot} \simeq 1 / 50$.

Send offprint requests to: S. A. Levshakov,

e-mail: sergei@oat.ts.astro.it

${ }^{3}(\mathrm{X} / \mathrm{H})$ is the logarithmic value of the element ratio by number, without reference to the solar value.

${ }^{4}[\mathrm{X} / \mathrm{H}]=(\mathrm{X} / \mathrm{H})-(\mathrm{X} / \mathrm{H}) \odot$. For the solar abundances we adopt $(\mathrm{O} / \mathrm{H})_{\odot}=-3.13 \pm 0.07,(\mathrm{Si} / \mathrm{H})_{\odot}=-4.44 \pm 0.01$, and $(\mathrm{Fe} / \mathrm{H})_{\odot}=-4.50 \pm 0.01$ from Grevesse et al. (1996) and the weighted average value $(\mathrm{Ar} / \mathrm{H})_{\odot}=-5.48 \pm 0.04$ from Sofia \& Jenkins (1998).
The neutral gas in I $Z_{w} 18$ has been observed in the 21-cm line (see van Zee et al. 1998 and references cited therein) as well as in several UV lines (Kunth et al. 1994; Pettini \& Lipman 1995; Vidal-Madjar et al. 2000). High velocity and high spatial resolution radio observations have shown that the overall kinematics of the $\mathrm{HI}$ gas associated with I Zw 18 is very complex and that the neutral gas velocity dispersion $\sigma$ equals $13-14 \mathrm{~km} \mathrm{~s}^{-1}$, or $b \equiv \sqrt{2} \sigma=18-20 \mathrm{~km} \mathrm{~s}^{-1}$ (van Zee et al. 1998). The H I column density in front of the bright star clusters in I Zw 18 deduced from the Ly- $\alpha$ absorption profile by Kunth et al., $N_{\mathrm{H}}=(3.5 \pm 0.5) \times 10^{21} \mathrm{~cm}^{-2}$, is comparable to the peak H I surface density found by van Zee et al., $N_{\mathrm{H} \text { I }} \simeq 3.0 \times 10^{21} \mathrm{~cm}^{-2}$. This high value is such that the neutral gas in I $\mathrm{Zw} 18$ can be considered as a local damped Ly- $\alpha$ system (DLA), similar to high-redshift DLAs observed in the light of many background quasars.

The first HST measurement of the $(\mathrm{O} / \mathrm{H})$ abundance in the $\mathrm{H}$ I region has indicated a possible discrepancy between the metal content in the neutral gas and in the $\mathrm{H}$ II regions (Kunth et al. 1994). This, however, was not confirmed in later studies by Pettini \& Lipman (1995) and by van Zee et al. (1998) who have shown that both the neutral and ionized gas in I Zw 18 may have the same oxygen abundance. 
New observations carried out with the Far Ultraviolet Spectroscopic Explorer (FUSE) by Vidal-Madjar et al. (2000) produce a similar puzzle: the column density ratios deduced from Ni, ArI, SiII, and Fe II lines in the neutral gas by using the usual Voigt-profile fitting procedure differ significantly from those derived for the H II regions by Izotov et al. (2001). Izotov et al. suggested that ionization in DLAs may affect abundance ratios. To interpret the observations, they proposed a model consisting of two regions with substantially different metal contents, i.e. implicitly recalling an idea of the existence of two media in BCGs (a pristine gas, unprocessed since the big bang, and a gas in the $\mathrm{H}$ II regions recently polluted by nucleosynthetic products - Kunth \& Sargent 1986). The discovery of such a primordial gas would be of great importance for cosmology, as noted by Kunth et al. (1994).

In this paper, we discuss the interpretation of the Ar I $\lambda 1048$, Si II $\lambda 1020$ and Fe II $\lambda 1063$ profiles of I Zw 18 published by Vidal-Madjar et al. (2000). We show that one can obtain - within the observational errors - the same metal content in the $\mathrm{H}$ II and $\mathrm{H}$ I regions, if one uses a generalized radiative transfer theory.

We also found that the observations are consistent with the $\alpha$-chain elements ( $\mathrm{Si}$ and $\mathrm{Ar}$ ) having the same $[\alpha$ chain/iron-peak] ratio as in halo stars with low metallicity.

\section{Data analysis and results}

The spectroscopic observations of I Zw 18 with FUSE in the range $\sim 980-1187 \AA$ are described in detail by VidalMadjar et al. (2000). The spectrum was obtained with a resolution of about $\lambda / \Delta \lambda \sim 10000$ and a signal-to-noise ratio of $S / N \sim 10$ per resolution element. The large entrance aperture $\left(30^{\prime \prime} \times 30^{\prime \prime}\right)$ fully covers the region containing the young stellar clusters $\left(\sim 10^{\prime \prime} \times 4^{\prime \prime}\right)$.

As noted by Vidal-Madjar et al., FUSE measures an average absorption over the full extent of the background UV sources. In this case the analysis of saturated absorption lines is not an easy and unambiguous task. The main difficulty is connected with the line broadening by possible large scale irregular (stochastic) velocity fields.

The influence of a finite correlation length of the random velocity field on the line profile depends on the details of the observation. If one considers the line formation process in front of a point source, then the observed spectrum reflects only one realization of the velocity field. Large deviations from the expectation value of the intensity $\left\langle I_{\lambda}\right\rangle$ may occur if the correlation length $\ell$ of the velocity field is not very small compared to the size of the absorbing region (Levshakov \& Kegel 1997). If, however, the spectrograph aperture covers an essential part of the galactic surface, we average over many individual lines of sight and therefore the expectation value $\left\langle I_{\lambda}\right\rangle$ should reasonably well correspond to the observations. In this case the standard Voigt-fitting analysis (based on the assumption of microturbulence) may yield misleading column densities. Namely, if correlation effects are important, saturated

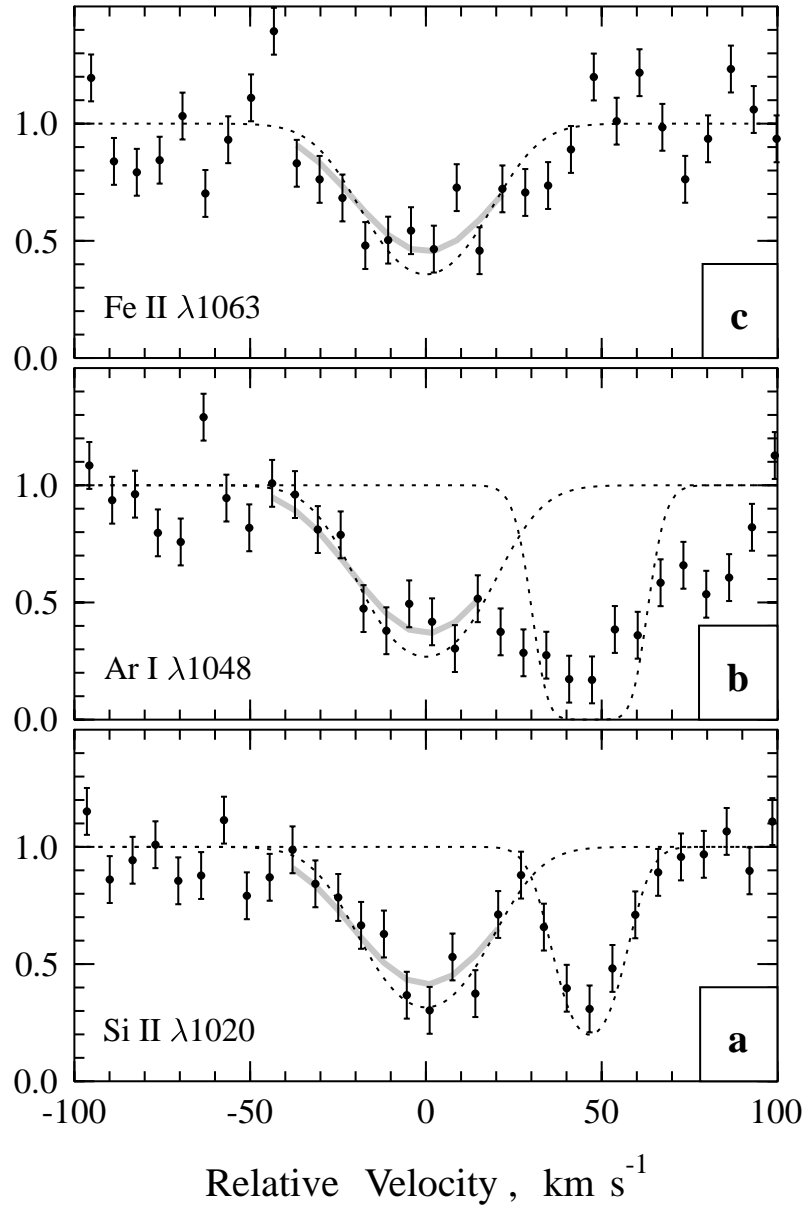

Fig. 1. Normalized intensities (dots with error bars) vs. radial velocities from FUSE spectra obtained by Vidal-Madjar et al. (2000). The grey lines are those parts of the synthetic spectra convolved with the spectrograph function which are used in our analysis. The dashed lines show the corresponding unconvolved spectra. Components at $\Delta v \simeq 49 \mathrm{~km} \mathrm{~s}^{-1}$ are the Galactic $\mathrm{H}_{2}$ lines: $\mathrm{L}(7-0) \mathrm{P}(4)$ in panel $\mathbf{a})$, and $\mathrm{L}(4-0) \mathrm{P}(1)$ in panel $\mathbf{b})$.

lines may appear to be unsaturated (Gail et al. 1974; Levshakov \& Kegel 1994).

Below we reanalyze the published FUSE data accounting for a finite correlation length of the velocity field.

\subsection{A microturbulent approach}

For comparison, we begin with the standard Voigt-profile fitting analysis which assumes a completely uncorrelated velocity field, i.e. $\ell=0$ (microturbulence). We use the published data from Fig. 1 of Vidal-Madjar et al. to illustrate how the column densities deduced from the Ar I, Si II and Fe II lines are affected by the underlying model assumptions. From the published data given in arbitrary units we have calculated normalized intensities using the plotted synthetic profiles, and then added to the normalized data $1 \sigma$ error bars corresponding to a $S / N=10$ as quoted by these authors (dots and error bars in Fig. 1). The profiles were centered at $v=0 \mathrm{~km} \mathrm{~s}^{-1}$ according to the synthetic spectra of Vidal-Madjar et al. The internal 
uncertainty of our velocity scale calibration was estimated to be about $\pm 5 \mathrm{~km} \mathrm{~s}^{-1}$.

The oscillator strengths of the Ar I $\lambda 1048.2199 \AA$, Si II $\lambda 1020.6989 \AA$ and Fe II $\lambda 1063.1764 \AA$ lines, $f_{\text {Ar I }}=$ $0.257, f_{\mathrm{Si} \text { II }}=0.01391$ and $f_{\mathrm{Fe} \text { II }}=0.0547$, were taken from Federman et al. (1992), Charro \& Martín (2000) and Raassen \& Uylings (1998), respectively, and their wavelengths from Morton (1991).

The absorption lines of Ar I and Si II are partly blended with the Galactic $\mathrm{H}_{2} \mathrm{~L}(4-0) \mathrm{P}(1)$ and $\mathrm{L}(7-0) \mathrm{P}(4)$ lines, respectively. Initially we fitted two component Voigt profiles to their observed intensities in order to estimate the influence of the $\mathrm{H}_{2}$ blends on the derived physical parameters. Their influence was found to be negligible (see Figs. $1 \mathrm{a}$ and $1 \mathrm{~b}$ where the $\mathrm{H}_{2}$ calculated profiles are shown by dotted curves). We therefore used in the following a one-component model and fitted all three metal lines (Ar I, Si II, and Fe II) simultaneously. In this procedure the theoretical profiles were convolved with the instrumental point-spread function (PSF) which we approximated by a Gaussian with the width of $26 \mathrm{~km} \mathrm{~s}^{-1}$. The best fit with $\chi_{\min }^{2}=24.76(M=30$ data points, and $\nu=26$ degrees of freedom) is shown in Fig. 1 by grey solid curves which also mark the data points involved in the optimization procedure. The dashed curves in Fig. 1 show the corresponding unconvolved profiles.

The estimated best fit parameters are: $b=(20.5 \pm$ $2.5) \mathrm{km} \mathrm{s}^{-1}, \log N_{\mathrm{Si} \text { II }}=15.05 \pm 0.05 \mathrm{~cm}^{-2}, \log N_{\mathrm{Ar} \text { I }}=$ $13.82 \pm 0.05 \mathrm{~cm}^{-2}$, and $\log N_{\text {Fe II }}=14.34 \pm 0.05 \mathrm{~cm}^{-2}$. This results in $(\mathrm{Ar} / \mathrm{Si})=-1.23 \pm 0.07$ and $(\mathrm{Fe} / \mathrm{Si})=$ $-0.71 \pm 0.07$ which agree quite well with the results of the Voigt-profile fitting analysis of Vidal-Madjar as quoted by Izotov et al. (2001), supporting our approximation of the PSF.

The most likely value for $b, 20.5 \pm 2.5 \mathrm{~km} \mathrm{~s}^{-1}$, is in good agreement with the radio observations of the $\mathrm{H}$ I gas in I Zw 18 carried out by van Zee et al. (1998). The fact that $b_{\mathrm{H} \text { I }} \simeq b_{\mathrm{Fe} \text { II }}$ implies that the kinetic temperature of the neutral gas in I Zw 18 is less than $500 \mathrm{~K}$ and that the line broadening is caused mainly by turbulent motions.

The estimated column densities, together with the $N_{\mathrm{H} \text { I }}$ from Kunth et al., imply however very low metal abundances, $[\mathrm{Si} / \mathrm{H}]=-2.05 \pm 0.09,[\mathrm{Ar} / \mathrm{H}]=-2.24 \pm 0.09$, and $[\mathrm{Fe} / \mathrm{H}]=-2.70 \pm 0.09$, as compared with $[\mathrm{O} / \mathrm{H}] \simeq-1.7$, measured previously by Izotov et al. (1999) in the H II regions.

With these data and $(\mathrm{O} / \mathrm{H})=-4.83 \pm 0.03$ (Izotov et al. 1999), we find $(\mathrm{Ar} / \mathrm{O})=-2.89 \pm 0.09$ and $[\mathrm{Ar} / \mathrm{O}]=$ $-0.54 \pm 0.12$, whereas nucleosythesis theory predicts for metal-poor gas $(\mathrm{Ar} / \mathrm{O}) \simeq-2.4$ (see, e.g., Woosley \& Weaver 1995; Limongi et al. 2000 and references cited therein). The observations of BCGs show $(\mathrm{Ar} / \mathrm{O})=$ $-2.25 \pm 0.09$ (Izotov \& Thuan 1999), or $(\mathrm{Ar} / \mathrm{O})=-2.32 \pm$ 0.05 (Kniazev et al. 2000). As for the abundance ratio $[\mathrm{Ar} / \mathrm{O}]$, it is found to be constant $(\simeq 0)$, independent of metallicity over about two decades of oxygen abundances (Henry \& Worthey 1999).
Thus, we can conclude that the microturbulent approach leads to abundance ratios which are difficult to reconcile with the theory of nucleosynthesis and with other observations. This fact indicates that a more general analysis of the observational data may be appropriate.

\subsection{A mesoturbulent approach}

21-cm observations with high spatial resolution show that the line profile varies with position (van Zee et al. 1998). This is a clear indication that large-scale motions (i.e. hydrodynamic flows with large correlation lengths) determine the line profiles, implying that the microturbulent approach is not well founded. We therefore generalize our analysis to include the effects of a finite correlation length, i.e. $\ell \neq 0$ (mesoturbulence) and to study also the limiting case $L / \ell \rightarrow 0$ (macroturbulence). We do this within the framework of the theory developed by Gail et al. (1974), i.e. we derive the intensity using the generalized transfer equation

$$
\begin{aligned}
\frac{\partial}{\partial s} q_{\lambda}(s, u)= & \frac{1}{\ell}\left[\frac{\partial^{2}}{\partial u^{2}} q_{\lambda}(s, u)-u \frac{\partial}{\partial u} q_{\lambda}(s, u)\right] \\
& -\kappa_{\lambda}(s, u) q_{\lambda}(s, u)
\end{aligned}
$$

where $q_{\lambda}(s, u)$ is the conditional intensity, $\kappa_{\lambda}(s, u)$ the local absorption coefficient, $s$ the coordinate along the line of sight, $u=v_{\|} / \sigma$ the normalized velocity parallel to the line of sight, and $\sigma$ the rms turbulent velocity.

Equation (1) was deduced under the assumption that the turbulent velocity field may be characterized by a Gaussian one point distribution function

$P_{1}(u)=\frac{1}{\sqrt{2 \pi}} \exp \left(-u^{2} / 2\right)$

and an exponential two point correlation function

$f(\Delta s)=\exp (-|\Delta s| / \ell)$,

with $\ell$ being the correlation length.

Once the conditional intensity $q_{\lambda}(s, u)$ has been determined, the expectation value of the ordinary intensity at the edge of the cloud $(s=L)$ follows from the relation

$\left\langle I_{\lambda}(s=L)\right\rangle=\int_{-\infty}^{\infty} q_{\lambda}(s=L, u) P_{1}(u) \mathrm{d} u$.

For more details we refer to the papers by Gail et al. (1974) and Levshakov \& Kegel (1994).

The simulation of the mesoturbulent Ar I, Si II and Fe II profiles has been carried out using the simplified model of a plane-parallel slab of geometrical size $L$ with homogeneous turbulence and uniform kinetic temperature, $T_{\text {kin }}$. Specifying the Ar I, Si II and Fe II column densities, the $L / \ell$ ratio, the velocity dispersion $\sigma$, and the thermal widths for each line, we can calculate the expectation value of the absorption-line profiles using the generalized radiative transfer Eq. (1).

The same $\chi^{2}$-minimization procedure as before was applied to the same data set, but now with three fitting parameters $\left\{N_{\mathrm{Ar} \text { I }}, N_{\mathrm{Si} \text { II }}, N_{\mathrm{Fe} \text { II }}\right\}$, i.e. $\nu=27$. We fixed 


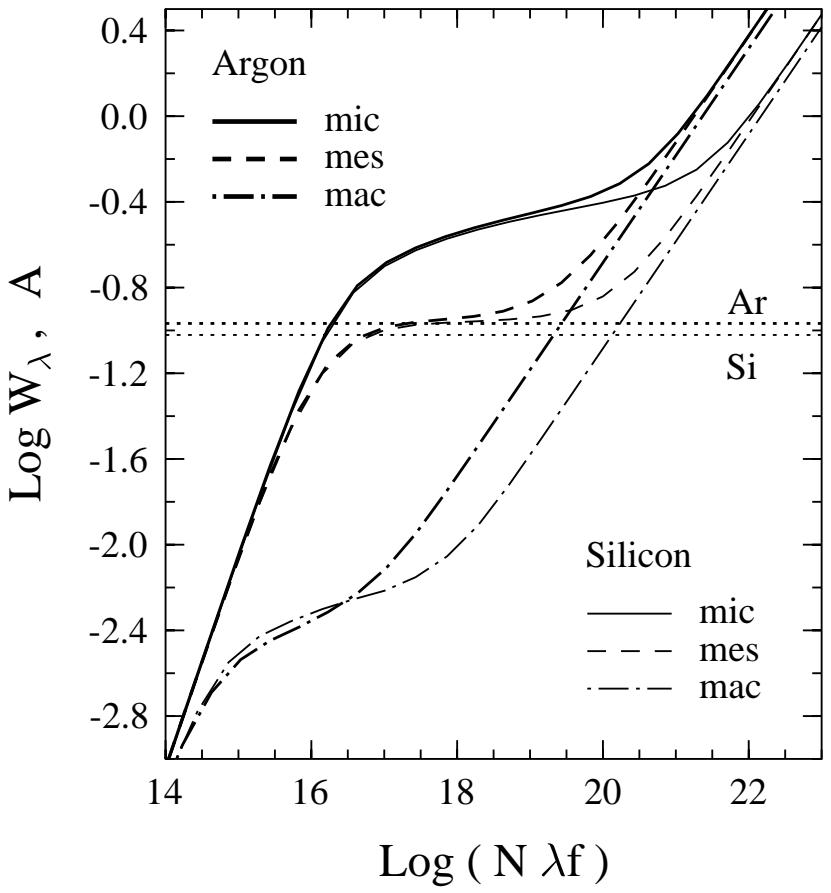

Fig. 2. Curves of growth for Ar I $\lambda 1048$ and Si II $\lambda 1020$ calculated for $b=18 \mathrm{~km} \mathrm{~s}^{-1}$ and $T_{\text {kin }}=200 \mathrm{~K}$. Three types of lines correspond to micro-, meso-, and macroturbulence $(\ell=0$, $L / \ell=1.45, L / \ell=0$, respectively). The dotted horizontal lines denote the estimated mean values of the Ar I and Si II equivalent widths.

$\sigma=14.5 \mathrm{~km} \mathrm{~s}^{-1}$ and $T_{\text {kin }}=200 \mathrm{~K}$ (i.e. $b \simeq 20.5 \mathrm{~km} \mathrm{~s}^{-1}$ ) and calculated $\chi_{\min }^{2}$ for a given $L / \ell$. The results are presented in Table 1. It should be emphasized that all Ar I, Si II and Fe II profiles corresponding to the listed - very different - solutions are almost identical to those shown in Fig. 1. (For all solutions we find the corresponding $\frac{1}{\nu} \chi_{\min }^{2}$ values to be smaller than 1.0.)

Our numerical results show that the derived column densities, as well as the abundance ratios, are strongly affected when correlation effects are accounted for. These effects are closely related to the changes in the curves of growth caused by the finite value of $L / \ell$. More accurately, the curve of growth depends on the value of $\tau_{\ell}$, the optical depth of a layer having the thickness of one correlation length. This implies that the shape of the curve of growth depends on the $f$-value of the line under consideration. As an example, Fig. 2 shows three curves of growth for the Ar I and Si II lines. They correspond to the two limiting cases of micro- and macroturbulence (Gail et al. 1974), as well as to an intermediate - mesoturbulent - case $(L / \ell=1.45)$. In general, one may say that the column density derived from a measured equivalent width increases with increasing correlation length $\ell$. Figure 2 also allows a qualitative interpretation of the $(\mathrm{Ar} / \mathrm{Si})$ ratio as a function of $L / \ell$ (see Table 1 and Fig. 3). In the microturbulent limit, both lines are still close to the linear part of the curve of growth, while in the mesoturbulent regime they lie on the flat part. Since the slope here is smaller than on the linear part, the $(\mathrm{Ar} / \mathrm{Si})$

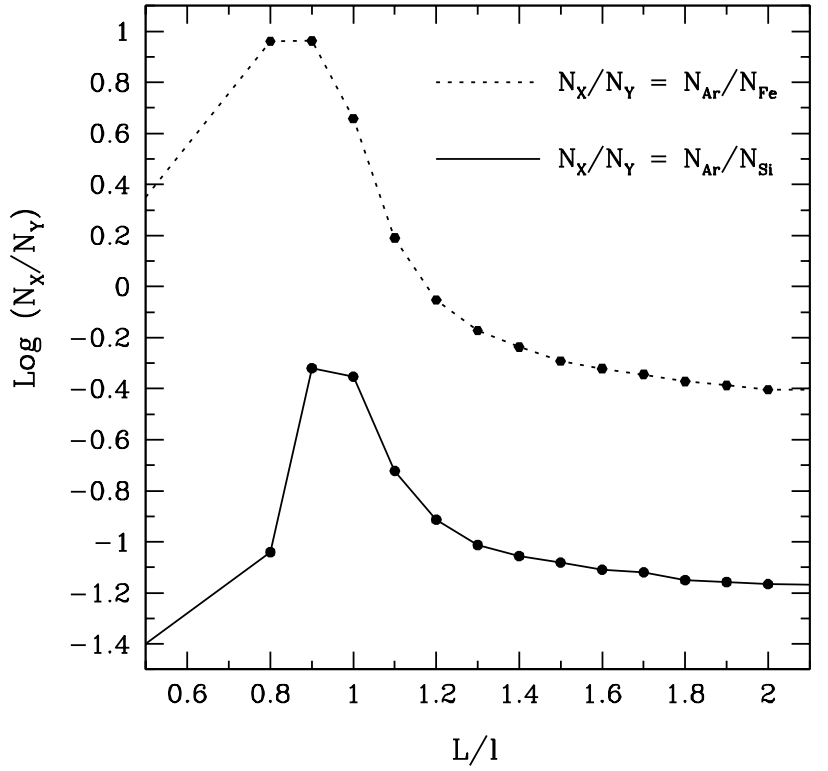

Fig. 3. Abundance ratios as functions of $L / \ell$ which is the ratio of the geometrical size of the absorbing region $L$ to the correlation length of the velocity field $\ell$. The data illustrate results presented in Table 1.

ratio is larger. In the macroturbulent limit, both lines lie on the square root part, i.e. their equivalent widths are dominated by radiation damping. In this case the slope is again steeper and, more importantly, the two curves of growth are well separated from each other (due to the different damping constants), implying a lower ( $\mathrm{Ar} / \mathrm{Si}$ ) ratio than in the mesoturbulent case. Thus, going from microto macroturbulence we expect the $(\mathrm{Ar} / \mathrm{Si})$ ratio at first to rise and, after going through a maximum, to decline again. From Fig. 2 we also see that in the macroturbulent limit the $(\mathrm{Ar} / \mathrm{Si})$ ratio is lower than in the microturbulent one. Since the Fe line is weaker, it is less affected by the correlation effects (see Table 1). Figure 3 also shows the ( $\mathrm{Ar} / \mathrm{Fe})$ ratio as function of $L / \ell$. The variation is dominated by that of the argon abundance.

From the FUSE data shown in Fig. 1 alone, it is not possible to determine the abundances or the abundance ratios with an acceptable accuracy. For this, additional information is necessary. For a purely empirical abundance determination, the measurement of more lines, in particular of several lines of the same ion having different $f$-values, are required. An alternative is to make further model assumptions and/or refer to nucleosynthesis theory in order to fix certain abundance ratios. We shall follow the latter approach for a consistent analysis.

For this we assume in the following that the argon abundance in the H I gas is the same as in the H II gas. (We choose argon since among the three elements under consideration its abundance is the best determined one in the HiI gas.) Izotov et al. (1999) obtained $(\mathrm{Ar} / \mathrm{H})=$ $-6.90 \pm 0.05(\mathrm{NW})$ and a slightly lower ratio for the SE region, $(\mathrm{Ar} / \mathrm{H})=-7.06 \pm 0.05$. This assumption restricts the parameter $L / \ell$ to the range 1.2 to 1.6 . 
The $\alpha$-chain elements $\mathrm{O}, \mathrm{Ar}$ and $\mathrm{Si}$ are produced in the same massive stars. Therefore their abundance ratio should not depend on the metallicity, a conclusion supported by observation (Izotov \& Thuan 1999). If we assume for $(\mathrm{Ar} / \mathrm{O})$ and $(\mathrm{Ar} / \mathrm{Si})$ in I $\mathrm{Zw} 18$ the solar values, then $L / \ell \approx 1.4-1.5$. For instance, if we assume $L / \ell=1.4$, we find from Table $1[\mathrm{Ar} / \mathrm{Si}]=-0.02 \pm 0.08$. With the value $(\mathrm{O} / \mathrm{H})=-4.83$ from Izotov et al. (1999), we find in addition $[\mathrm{Ar} / \mathrm{O}]=0.10 \pm 0.12$. For $L / \ell=1.5$ we find correspondingly $[\mathrm{Ar} / \mathrm{Si}]=-0.05 \pm 0.08$, and $[\mathrm{Ar} / \mathrm{O}]=$ $0.02 \pm 0.12$.

We carried out similar calculations also with two limiting values of the broadening parameter, $b=18$ and $23 \mathrm{~km} \mathrm{~s}^{-1}$, making the same additional assumptions as before. The mean values for $L / \ell=1.4$ are as follows: $[\mathrm{Fe} / \mathrm{H}]=-2.4 \pm 0.1$, and $[\mathrm{Si}, \mathrm{Ar} / \mathrm{Fe}]=0.7 \pm 0.1$.

To conclude, we note that the selected solution $(L / \ell=$ 1.4) yields $(\mathrm{Ar} / \mathrm{Si})=-1.06 \pm 0.08$ and $(\mathrm{Fe} / \mathrm{Si})=-0.81 \pm$ 0.09 . These values differ from those derived for the H II regions of $\mathrm{I} \mathrm{Zw} 18:(\mathrm{Ar} / \mathrm{Si})=-0.75 \pm 0.23$ and $(\mathrm{Fe} / \mathrm{Si})=$ $-0.15 \pm 0.25$ (SE) (see Izotov \& Thuan 1999; and Izotov et al. 1999). This discrepancy, however, can hardly be considered as a serious argument against our assumption that the metal abundances in the neutral gas are the same as in the ionized gas, since the measurement of the Si abundance in the SE emission patch is not very accurate due to the non-detection of the Si III] $\lambda 1892$ emission line which is predicted by atomic theory "given the signal-to-noise ratio and intensity of the neighboring Si III $] \lambda 1883$ emission line" (Izotov \& Thuan 1999). The Fe abundance measurements in the $\mathrm{HII}$ regions of I $\mathrm{Zw} 18$ are also not precise. One cannot exclude some "contribution of C IV $\lambda 4658$ emission line to a weak [Fe III] $\lambda 4658$ line. In this case observations of $\mathrm{H}$ II regions may overestimate the Fe abundance" (Izotov 2000, private communication). Indeed, the ratio $[\mathrm{Fe} / \mathrm{H}]=-1.96 \pm 0.09$ found by Izotov et al. (1999) is about 3 times larger than the value we estimated for the $\mathrm{H}$ I gas. Taking these results at their face values, the SE $\mathrm{H}$ II region yields the following $[\alpha$-chain/iron-peak $]$ ratios: $[\mathrm{Ar} / \mathrm{Fe}]=0.38 \pm 0.11,[\mathrm{Si} / \mathrm{Fe}]=0.09 \pm 0.25$, and $[\mathrm{O} / \mathrm{Fe}]$ $=0.27 \pm 0.11$, which are systematically lower than the mesoturbulent results we obtained with $L / \ell=1.4$ for the neutral gas.

\section{Discussion}

The history of star formation in galaxies is closely connected with a gradual chemical enrichment of the interstellar gas because the rate of heavy element production in stars is higher than the destruction rate (e.g. Samland 1998). During production processes, heavy elements have different nucleosynthetic histories so that their relative abundances are not expected to remain constant in time. For instance, galactic chemical evolution models predict an overabundance of the $\alpha$-chain elements relative to the iron-peak elements in the metal-poor gas (e.g. Timmes et al. 1995). The $[\alpha / \mathrm{Fe}]$ ratio decreases with increasing global metallicity as characterized by the $[\mathrm{Fe} / \mathrm{H}]$ ratio.
This ratio represents a sort of chronometer due to the continuous increase of iron in the ISM (e.g. Wheeler et al. 1989).

Oxygen, silicon, argon and other $\alpha$-chain elements are typical products of Type II SNe, while iron is also produced by Type Ia SNe which have longer evolution scales. A time delay of $\sim 0.5-1$ Gyr between the production of the $\alpha$-elements in Type II SNe and the iron-peak elements in Type Ia SNe is usually assumed (e.g. Pettini 2000). Therefore, in the early stages of chemical enrichment the $\alpha$-elements are naturally expected to be relatively more abundant than the iron-peak elements. Our estimate of the metal abundances in the $\mathrm{HI}$ gas of I $\mathrm{Zw} 18$ is consistent with this picture and yields similar abundance ratios as observed in halo stars with low metallicity. For instance, Israelian et al. (1998) found for halo stars with $-2.0 \geq$ $[\mathrm{Fe} / \mathrm{H}] \geq-2.5$ the ratio $[\mathrm{O} / \mathrm{Fe}]$ to be in the range 0.3 to 1.0. Similar results were obtained by Gratton \& Sneden (1988) for the ratio $[\mathrm{Si} / \mathrm{Fe}]$.

The argon abundance ratio $[\mathrm{Ar} / \mathrm{Fe}]$ has not been determined directly in either dwarfs or field giants since Ar has no optical transitions from low excitation levels. However, theoretical calculations predict approximately the same $[\mathrm{Ar} / \mathrm{Fe}]$ ratio as for other $\alpha$-chain elements (Timmes et al. 1995).

It is interesting to compare our estimates of the metal abundances in I Zw 18 with those of two well-studied high redshift DLAs: the $z=3.390$ DLA towards Q0000-2620 where $[\mathrm{Fe} / \mathrm{H}] \simeq-2$ and $[\alpha$-chain/iron-peak $] \simeq 0.20$ were measured by Molaro et al. (2000, 2001), and the $z=3.386$ DLA towards $\mathrm{Q} 0201+1120$ with $[\mathrm{Fe} / \mathrm{H}] \simeq-1.4$ and $[\alpha-$ chain/iron-peak $] \simeq 0.16$ deduced by Ellison et al. (2001). Both high- $z$ DLAs do not show a pronounced enhancement of the $\alpha$-elements as compared with I Zw 18. The low $[\alpha$-chain/iron-peak] ratios in metal-poor DLAs have been interpreted as evidence for low or episodic star formation in these galaxies, - there should be sufficient time since the last burst of star formation to accumulate iron in the ISM to a near-solar value relative to $\alpha$-elements (e.g. Pettini et al. 2000; Centurión et al. 2000; Molaro et al. 2000).

In contrast, our estimates for the DLA system I Zw 18 at $z=0.0025$ indicate $[\alpha$-chain/iron-peak $] \simeq 0.7$. Thus, the nearby system I Zw 18 appears to be chemically younger than these high-redshift DLAs.

\section{Conclusions}

We have shown that, if correlations in the large-scale velocity field are accounted for, it is possible to derive metal abundances from the absorption profiles of $\operatorname{ArI} \lambda 1048$, Si II $\lambda 1020$, and Fe II $\lambda 1063$, observed in the spectrum of the damped Ly- $\alpha$ system I Zw 18 by Vidal-Madjar et al. (2000), that are consistent with the theory of nucleosynthesis and are similar to those derived from spectra of Galactic metal-poor stars and those derived for H II regions in metal-deficient BCGs. The fact that 21-cm observations with high spatial resolution show the presence of 
Table 1. Ar, Si and Fe abundances of neutral gas in I Zw 18, calculated with $b=20.5 \mathrm{~km} \mathrm{~s}^{-1}$ for different values of $L / \ell$.

\begin{tabular}{lccccccc}
\hline$L / \ell$ & $N_{\mathrm{Ar} \text { I }}^{a}, \mathrm{~cm}^{-2}$ & $N_{\mathrm{Si} \text { II }}^{a}, \mathrm{~cm}^{-2}$ & $N_{\mathrm{Fe} \text { II }}^{a}, \mathrm{~cm}^{-2}$ & $(\mathrm{Ar} / \mathrm{H})^{b}$ & $(\mathrm{Si} / \mathrm{H})^{b}$ & $(\mathrm{Fe} / \mathrm{H})^{b}$ & $(\mathrm{Ar} / \mathrm{Si})^{c}$ \\
\hline 0 & $1.2(17)$ & $1.2(19)$ & $5.6(17)$ & -4.45 & -2.48 & -3.79 & -1.97 \\
0.8 & $2.1(16)$ & $2.3(17)$ & $2.3(15)$ & -5.23 & -4.18 & -6.17 & -1.05 \\
0.9 & $1.1(16)$ & $2.3(16)$ & $1.2(15)$ & -5.49 & -5.18 & -6.46 & -0.31 \\
1.0 & $4.0(15)$ & $9.0(15)$ & $8.8(14)$ & -5.95 & -5.59 & -6.60 & -0.36 \\
1.1 & $1.1(15)$ & $5.8(15)$ & $7.1(14)$ & -6.48 & -5.78 & -6.69 & -0.71 \\
1.2 & $5.5(14)$ & $4.5(15)$ & $6.2(14)$ & -6.80 & -5.89 & -6.75 & -0.91 \\
1.3 & $3.7(14)$ & $3.8(15)$ & $5.5(14)$ & -6.97 & -5.97 & -6.80 & -1.00 \\
1.4 & $2.9(14)$ & $3.3(15)$ & $5.0(14)$ & -7.08 & -6.03 & -6.84 & -1.06 \\
1.5 & $2.4(14)$ & $2.9(15)$ & $4.7(14)$ & -7.16 & -6.07 & -6.87 & -1.09 \\
1.6 & $2.1(14)$ & $2.7(15)$ & $4.4(14)$ & -7.22 & -6.11 & -6.90 & -1.11 \\
1.7 & $1.9(14)$ & $2.5(15)$ & $4.2(14)$ & -7.27 & -6.14 & -6.92 & -1.13 \\
1.8 & $1.7(14)$ & $2.4(15)$ & $4.0(14)$ & -7.30 & -6.17 & -6.94 & -1.14 \\
1.9 & $1.6(14)$ & $2.3(15)$ & $3.9(14)$ & -7.34 & -6.19 & -6.96 & -1.15 \\
2.0 & $1.5(14)$ & $2.2(15)$ & $3.8(14)$ & -7.36 & -6.21 & -6.97 & -1.16 \\
microturbulence & & & & & & \\
$\infty$ & $6.7(13)$ & $1.1(15)$ & $2.2(14)$ & -7.72 & -6.50 & -7.20 & -1.23 \\
\hline
\end{tabular}

The internal accuracy is ${ }^{a} 0.05 \mathrm{dex},{ }^{b} 0.09 \mathrm{dex},{ }^{c} 0.07 \mathrm{dex} ; N_{\mathrm{H} \mathrm{I}}=3.5 \times 10^{21} \mathrm{~cm}^{-2}$ from Kunth et al. (1994) is adopted.

velocity fields with large correlation lengths, is a strong support for our mesoturbulent model. We note in particular, that the velocity dispersion determined for the neutral gas by means of a $\chi^{2}$-minimization procedure, is in good agreement with the $\mathrm{H}$ I $21-\mathrm{cm}$ observations.

If large-scale motions in the ISM are ubiquitous for all galaxies then we can expect that the correlation effects will be significant for abundance determinations whenever optical thickness effects become important. Our analysis shows that the standard Voigt-profile fitting procedure may severely underestimate column densities, especially for saturated lines.

We note in passing that the method used in the present paper, in which the expectation value of the intensity is determined from the generalized transfer equation (see Gail et al. 1974; Levshakov \& Kegel 1994), differs from that used for the interpretation of QSO spectra, the latter being a Monte Carlo method (Levshakov et al. 1998a, 1998b). In the present case the observations refer to the intensity averaged over many lines of sight, while in the case of QSO spectra we have only one line of sight.

The additional parameter introduced in the generalized radiative transfer equation, $L / \ell$, can, in principle, be estimated independently and, thus, can be used to check the criteria applied to select the appropriate solution from Table 1 . Namely, $L / \ell$ can be found from the analysis of saturated and unsaturated lines of the same ion, since the latter is less affected by the correlation effects (see Fig. 2). For our case, observations of the Ar I $\lambda 1066$ line would be of particular interest since its oscillator strength $f_{1066}=1 / 4 f_{1048}$. The $\operatorname{Ar}$ I $\lambda 1066$ line was observed in the $z=3.4$ DLA system toward the quasar Q0000-2620 (Molaro et al. 2001). The similarity of both DLA systems is also supported by the very low molecular hydrogen contents found in I $\mathrm{Zw} 18$, $f\left(\mathrm{H}_{2}\right) \equiv 2 N_{\mathrm{H}_{2}} / N_{\mathrm{H}_{\mathrm{I}}} \ll 10^{-6}$ (Vidal-Madjar et al. 2000), and at $z=3.4, f\left(\mathrm{H}_{2}\right)=(6.8 \pm 2.0) \times 10^{-8}$ (Levshakov et al. 2000, 2001).

Since Ar can be partially ionized by nearby UV stellar radiation with energies $h \nu>15.76 \mathrm{eV}$, the relative abundance of Ar I is a good indicator of the intensity of the local photoionizing flux (Sofia \& Jenkins 1998). Our measurements do not indicate the presence of any significant amount of partially ionized gas in the damped $\mathrm{L} \alpha$ system I Zw 18 and, hence, metal abundances in the neutral gas do not require ionization corrections as suggested by Izotov et al. (2001).

Thus, it is extremely important in future observations to investigate other absorption lines of neutral or singly ionized atoms in order to understand better the kinematic characteristics of the neutral gas bulk motion which will enable us to obtain more reliable estimations of metallicities in I Zw 18.

Acknowledgements. We thank Don Morton and Chris Howk for providing Fe II atomic data, Yuri Izotov for useful remarks on an early version of the paper, and our referee James Lequeux for helpful comments. The work of S.A.L. and I.I.A. is supported in part by the Deutsche Forschungsgemeinschaft and by the RFBR grant No. 00-02-16007.

\section{References}

Centurión, M., Bonifacio, P., Molaro, P., \& Vladilo, G. 2000, ApJ, 536, 540

Charro, E., \& Martín, I. 2000, ApJS, 126, 551

Ellison, S. L., Pettini, M., Steidel, C. C., \& Shapley, A. E. 2001, ApJ, 549, 770

Federman, S. R., Beideck, D. J., Schectman, R. M., \& York, D. G. 1992, ApJ, 401, 367

Gail, H. P., Hundt, E., Kegel, W. H., Schmid-Burgk, J., \& Traving, G. 1974, A\&A, 32, 65

Gratton, R. G., \& Sneden, C. 1988, A\&A, 204, 193

Grevesse, N., Noels, A., \& Sauval, A. J. 1996, in Cosmic Abundances, ASP Conf. Ser., 99, 117 
Henry, R. B. C., \& Worthey, G. 1999, PASP, 111, 919

Izotov, Yu. I., \& Thuan, T. X. 1999, ApJ, 511, 639

Izotov, Yu. I., Chaffee, F. H., Foltz, C. B., et al. 1999, ApJ, 527,757

Izotov, Yu. I., Schaerer, D., \& Charbonnel, C. 2001, ApJ, 549, 878

Izraelian, G., García López, R. G., \& Rebolo, R. 1998, ApJ, 507,805

Kniazev, A. Yu., Pustilnik, S. A., Ugryumov, A. V., \& Kniazeva, T. F. 2000, Astr. Lett., 26, 129

Kunth, D., \& Sargent, W. L. W. 1986, ApJ, 300, 496

Kunth, D., Lequeux, J., Sargent, W. L. W., \& Viallefond, F. 1994, A\&A, 282, 709

Limongi, M., Straniero, O., \& Chieffi, A. 2000, ApJS, 129, 625

Levshakov, S. A., \& Kegel, W. H. 1994, MNRAS, 271, 161

Levshakov, S. A., \& Kegel, W. H. 1997, MNRAS, 288, 787

Levshakov, S. A., Kegel, W. H., \& Takahara, F. 1998a, ApJ, 499, L1

Levshakov, S. A., Kegel, W. H., \& Takahara, F. 1998b, A\&A, 336, L29

Levshakov, S. A., Molaro, P., Centurión, M., et al. 2000, A\&A, 361,803

Levshakov, S. A., Molaro, P., Centurión, M., et al. 2001, in Proc. ESO/ST-ECF/STScI Workshop, Deep Fields [astro-ph/0011470]
Molaro, P., Bonifacio, P., Centurión, M., et al. 2000, ApJ, 541, 54

Molaro, P., Levshakov, S. A., D’Odorico, S., Bonifacio, P., \& Centurión, M. 2001, ApJ, 549, 90

Morton, D. C. 1991, ApJS, 77, 119

Östlin, G. 2000, ApJ, 535, L99

Pettini, M. 2000, Phil. Trans. R. Soc. London, Ser. A, 358, 2035 [astro-ph/0001075]

Pettini, M, \& Lipman, K. 1995, A\&A, 297, L63

Pettini, M, Ellison, S. L., Steidel, C. C., Shapley, A. E., \& Bowen, D. V. 2000, ApJ, 532, 65

Raassen, A. J. J., \& Uylings, P. H. M. 1998, J. Phys. B, 31, 3137

Samland, M. 1998, ApJ, 496, 155

Sofia, U. J., \& Jenkins, E. B. 1998, ApJ, 499, 951

Timmes, F. X., Woosley, S. E., \& Weaver, T. A. 1995, ApJS, 98,617

van Zee, L., Westpfahl, D., Haynes, M. P., \& Salzer, J. J. 1998, AJ, 115,1000

Vidal-Madjar, A., Kunth, D., Lecavelier des Etangs, A., et al. 2000, ApJ, 538, L77

Wheeler, J. C., Sneden, C., \& Truran, J. W. 1989, ARA\&A, 27, 279

Woosley, S. E., \& Weaver, T. A. 1995, ApJS, 101, 181

Zwicky, F. 1966, ApJ, 143, 192 